\title{
Evaluación anatómica e histopatológica de la anastomosis yeyunal equina revestida con xenoinjerto de pericardio bovino
}

\author{
Anatomical and histopathological evaluation of the equine jejunal anastomosis \\ coated with bovine pericardium xenograft
}

\author{
Ceesar N. Aguilar G. ${ }^{1}$, Alfredo Delgado C. ${ }^{1}$, Alfonso Chavera C. ${ }^{2}$, Medalí Cueva R. ${ }^{1}$
}

\section{Resumen}

Se hizo la evaluación del xenoinjerto de pericardio bovino no tratado como biomaterial para el revestimiento de la anastomosis de intestino delgado en equinos. Se practicó la cirugía a siete equinos con edades entre 3 y 10 años. Se seleccionaron al azar a dos de ellos, haciendo una laparatomía a uno de ellos a los 15 días y al otro a los 100 días del posoperatorio. En los estudios macroscópicos e histopatológicos de la zona cicatricial de la anastomosis término-terminal se evidenció que el xenoinjerto no se contrae ni se desplaza de la línea de sutura, garantizando el proceso de remodelación cicatricial y evitando complicaciones como peritonitis, estenosis, abscesos, fugas anastomóticas y adherencias. Se concluye que el xenoinjerto de pericardio bovino no tratado es viable y efectivo, como revestimiento de la enterectomía yeyunal; demostrando buena adaptabilidad como microambiente natural para células progenitoras encargadas de la remodelación cicatricial, aunque conlleva a una moderada flogosis y fibrosis que después del periodo de convalecencia desaparecen progresivamente, sin observarse calcificación ni degeneración del colágeno.

Palabras clave: equino; pericardio bovino; anastomosis; xenoinjerto; enterectomía; intestino delgado; biomaterial

\section{AbSTRaCT}

The evaluation of the untreated bovine pericardium xenograft as a biomaterial for the lining of the small intestine anastomosis in equines was conducted. Seven horses with ages between 3 and 10 years were operated on. Two of them were randomly selected, doing a laparotomy to one of them at 15 days and the other at 100 days post-surgery. The

1 Clínica de Animales Mayores, Facultad de Medicina Veterinaria, Universidad Nacional Mayor de San Marcos, Lima, Perú

2 Laboratorio de Histopatología, Embriología y Patología Animal, Facultad de Medicina Veterinaria, Universidad Nacional Mayor de San Marcos, Lima, Perú

3 E-mail: ceniague@gmail.com

Recibido: 30 de enero de 2018

Aceptado para publicación: 26 de julio de 2018 
macroscopic and histopathological evaluation of the end-to-end anastomosis cicatricial area evidenced that the xenograft does not contract or move from the line of suture, guaranteeing the process of cicatricial remodeling and avoiding complications such as peritonitis, stenosis, abscesses, anastomotic leakage and adhesions. It is concluded that the untreated bovine pericardium xenograft is viable and effective as a jejunal enterectomy lining, showing good adaptability as a natural microenvironment for progenitor cells responsible for cicatricial remodeling, although it leads to a moderate phlogosis and fibrosis that disappear progressively after the convalescence period, without calcification or collagen degeneration.

Key words: equine; bovine pericardium; anastomosis; xenograft; enterectomy; small intestine; biomaterial

\section{INTRODUCCIÓN}

La falla anastomótica gastrointestinal produce dehiscencia, fugas y fístulas, consideradas complicaciones importantes después de la cirugía a pesar de las mejoras en la técnica quirúrgica (Telem et al., 2010; Testini et al., 2014). Las adherencias frecuentemente causan frustración entre los profesionales de la salud, lo que estimula la investigación de productos y métodos para prevenir su formación (Claunch y Mueller, 2012), dado que repercuten en cólico posoperatorio, siendo la segunda complicación más común que conlleva a laparotomía repetida, con tasas de supervivencia reportadas entre 0 y $20 \%$ (Alonso et al., 2014).

Actualmente se disponen de parches de pericardio bovino tratados con glutaraldehído para evitar el trasplante de proteínas o ADN bovino en el receptor, que son usados generalmente en cirugía cardiovascular, pero que podrían calcificarse e incluso adquirir un comportamiento citotóxico (Li et al., 2014). El pericardio bovino tratado con glutaraldehído se ha usado, además, para parchar la línea de sutura de la anastomosis yeyunal, con miras a mejorar la técnica quirúrgica entérica en medicina humana (Testini et al., 2014).

El presente estudio tuvo como objetivo caracterizar macroscópica e histopatológicamente la zona quirúrgica de la anasto- mosis término-terminal con revestimiento de xenoinjerto de pericardio bovino sin tratar en dos equinos.

\section{Materiales y Métodos}

El presente estudio forma parte de un estudio más amplio, parte del cual ha sido reportado (Aguilar et al., 2018). El trabajo se hizo en las instalaciones de la Universidad Nacional de Cajamarca, Perú, bajo condiciones de campo, utilizándose para esta investigación siete equinos criollos (machos y hembras) entre 3 y 10 años, procedentes de la campiña de Cajamarca. Los animales fueron dosificados con Ivermectina al $1.85 \%$ y recibieron alfalfa ad libitum durante 20 días previos a la cirugía. Los caballos fueron clasificados como ASA 1, según los criterios de la Asociación Americana de Anestesiología (Nazar et al., 2015).

Los equinos fueron sometidos a enterectomía término-terminal aplicándose un xenoinjerto de pericardio bovino como revestimiento. La técnica anestésica y quirúrgica se encuentran descritas en Aguilar et al. (2018) (Ver Figura 1). En términos generales, se realizó una laparatomía ventral medial en la línea alba, a la altura del ombligo, de 8 a $12 \mathrm{~cm}$, se exteriorizó un asa intestinal yeyunal, se ligaron los vasos sanguíneos, se seccionó el asa yeyunal $(10 \mathrm{~cm})$ en corte oblicuo en un 
ángulo de $50^{\circ}$ (Freeman, 2012). La anastomosis término-terminal se realizó con un patrón de sutura simple discontinuo (Fossum, 2009), con material de sutura absorbible desde la serosa e incluyendo la muscular longitudinal, la muscular circular y la submucosa basados en las estructuras histológicas (Gelberg, 2014). El xenoinjerto de pericardio bovino no tratado fue aplicado según el reporte de Testini (2014), donde la línea de sutura fue revestida con el xenoinjerto utilizando de 8 a 10 suturas simples discontinuas para fijarlo en la línea de sutura de la anastomosis (Figura 1). El cierre del mesenterio se hizo con sutura simple continua (Bladon y Hillyer, 2000).
El xenoinjerto pericárdico fibroso se obtuvo de bovinos menores a 6 años de un centro de beneficio, antes de que las vísceras torácicas pierdan su condición de limpieza fisiológica y sin recibir tratamiento previo. El tejido fue trasportado en suero fisiológico para usarse en la línea de sutura anastomótica (ver Aguilar et al., 2018).

Dos de los siete equinos sometidos a la enterectomía yeyunal término-terminal revestida con xenoinjerto de pericardio bovino fueron seleccionados al azar para realizar la caracterización macroscópica e histopatológica de la línea de sutura quirúrgica, uno a los 15 días y el otro a los 100 días del posoperatorio.

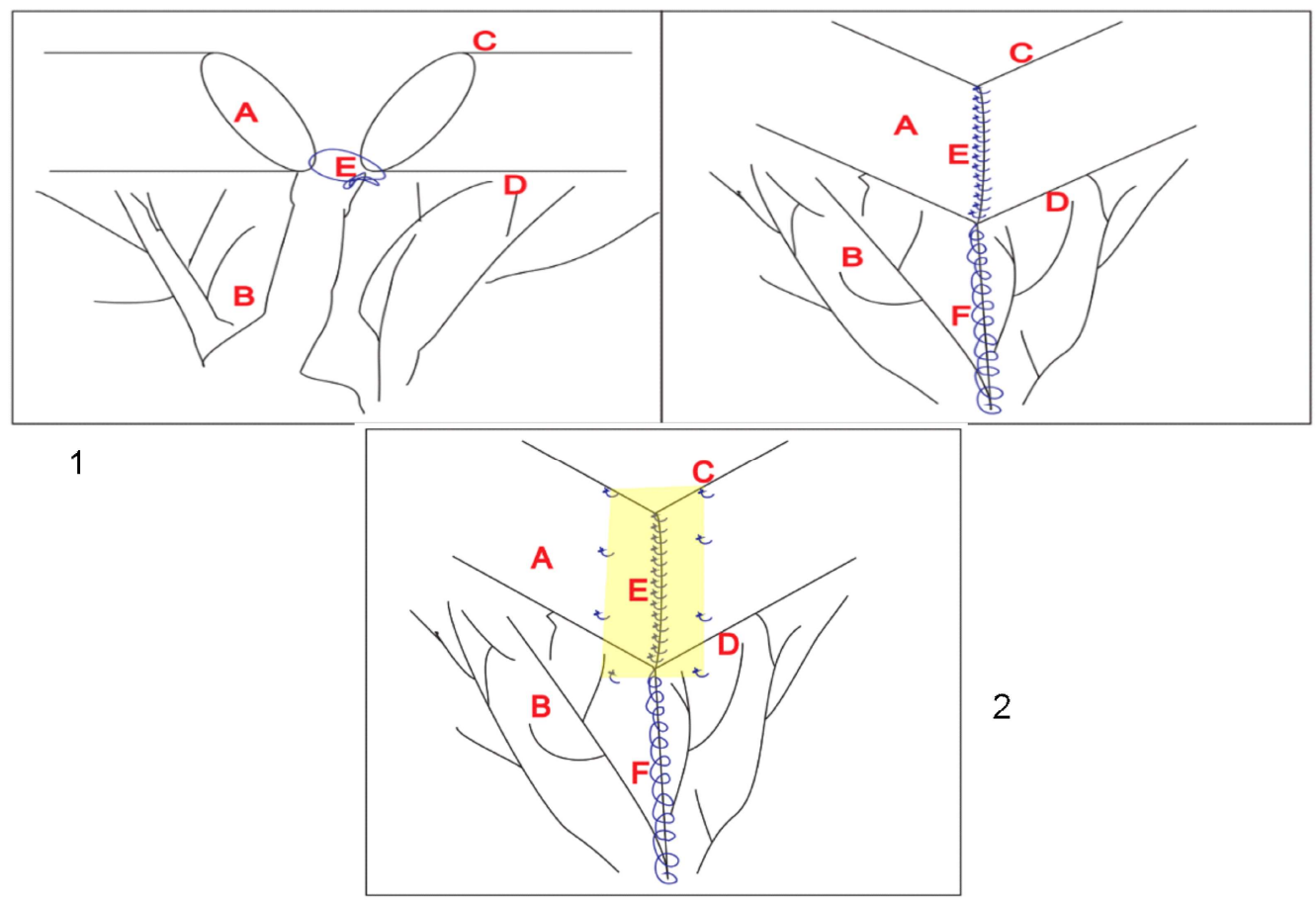

Figura 1. Ilustración del corte experimental del yeyuno en ángulo aproximado de $50^{\circ}$ para extraer el segmento intestinal y la colocación de las suturas de ácido poliglicólico. (a) Yeyuno; (b) Mesenterio; (c) Zona contramesentérica (termina en conformación de «V»); (d) Zona mesentérica; (e) Sutura simple discontinua en la línea anastomótica; (f) Sutura simple continua del mesenterio. 2. Ilustración del revestimiento con xenoinjerto de pericardio bovino sobre la línea de sutura anastomótica (color amarillo), fijado con suturas simples discontinuas de ácido poliglicólico. (a) Yeyuno; (b) Mesenterio; (c) Zona contramesentérica; (d) Zona mesentérica; (e) Sutura simple discontinua en la línea anastomótica; (f) Sutura simple continua del mesenterio (tomado de Aguilar et al., 2018) 
Para esto, se hizo una segunda laparotomía exploratoria a nivel de la línea alba, previo ayuno de 18 horas, con una incisión generada en la línea alba desde el xifoides hasta la zona inguinal, con la finalidad de exponer todo el contenido abdominal. Macroscópicamente se determinaron posibles estenosis, abscesos, fuga anastomótica, peritonitis, contracción o desplazamiento del xenoinjerto, dándoles un escore de 0 a 4 puntos $(0=$ ausente, $1=$ mínima, $2=$ moderada, $3=$ considerable, $4=$ severa); como también la presencia de adherencias con un score de 0 a 3 puntos $(0$ $=\sin$ adherencias; $1=$ adherencias ligeras; 2 $=$ adherencias fijas; $3=$ adherencias sólidas, solo removibles con daño) (Testini et al., 2014). Se registraron, además, las variaciones macroscópicas de color, movimientos peristálticos, determinando la actividad biomecánica al verificar el tránsito de los contenidos intraluminales (Sasaki y Yoshihara, 1998). Así mismo, se evaluó la presencia o ausencia macroscópica del hilo de sutura en la zona anastomótica (Figura 2).

Se tomó una sección de $4 \mathrm{~cm}$ anterior y $4 \mathrm{~cm}$ posterior a línea de sutura anastomótica con xenoinjerto de pericardio bovino para el análisis histopatológico. Las muestras se fijaron con formalina tamponada neutra y encajadas en parafina. Se hicieron cortes longitudinales en secciones consecutivas de $3 \mu \mathrm{m}$ de espesor (longitudinalmente al tracto intestinal), con el fin de observar la sección entera desde la mucosa hasta la serosa. Los cortes fueron teñidos con hematoxilina-eosina, según técnicas histopatológicas estándares. Se describieron los procesos de remodelación cicatricial y se evaluaron los grados de inflamación (flogosis), fibrosis, así como los estados de infiltración del xenoinjerto, de la mucosa y de la serosa, según la escala de Testini et al. (2014); además de evaluar la reacción histológica causada por el hilo de sutura.

El estudio fue aprobado por el Comité de Ética Institucional de la Unidad de Posgrado de la Facultad de Medicina Veterinaria de la Universidad Nacional Mayor de San Marcos. En la metodología se respeta- ron las directrices internacionales para la investigación biomédica respecto al uso de animales.

\section{Resultados}

Se evidenció un correcto proceso de cicatrización, destacando la ausencia de abscesos, peritonitis, fugas anastomóticas y estenosis que impidan el peristaltismo a los 15 días (Figura 3), presentando en todos los casos un grado 0 en la escala de Testini et al. (2014), lo que indica un adecuado funcionamiento del sistema nervioso entérico (Berthoud, 2008; Furness, 2008; Merritt y Julliand, 2013), que junto a las células intersticiales de Cajal generan los patrones motores que rigen la motilidad gastrointestinal (Koning y Liebich, 2011; Mazzone y Farrugia, 2007). Por otro lado, no se encontró contracción ni desplazamiento del xenoinjerto de pericardio bovino, ni adherencias al xenoinjerto, intestinos o a la pared abdominal (grado 0 en la escala de Testini et al. [2014]).

Al realizar pruebas de distención, se comprobó que el intestino normal podía llegar a distenderse mucho más que la zona anastomótica con xenoinjerto de pericardio bovino. Por otro lado, al evaluar el peristaltismo, la zona anastomótica evidenció un comportamiento similar al resto del intestino, destacando la acción inofensiva del xenoinjerto a la fisiología intestinal (Koenig y Cote, 2006). Así mismo, el hilo de sutura ácido poliglicólico 3-0 -, se veía sin cambios aparentes a los 15 días del posoperatorio y no evidenciándose a los 100 días. Finalmente, es importante señalar que los nódulos linfáticos mesentéricos no fueron evidentes a la palpación durante la relaparotomía.

En la evaluación histopatológica (Cuadro 1) se demostró que a los 15 días de colocado el xenoinjerto no se diferenciaba de la serosa intestinal, aunque contenía infiltración de neutrófilos, con tejido de granulación y angioblastos; sin embargo, se encontró una 

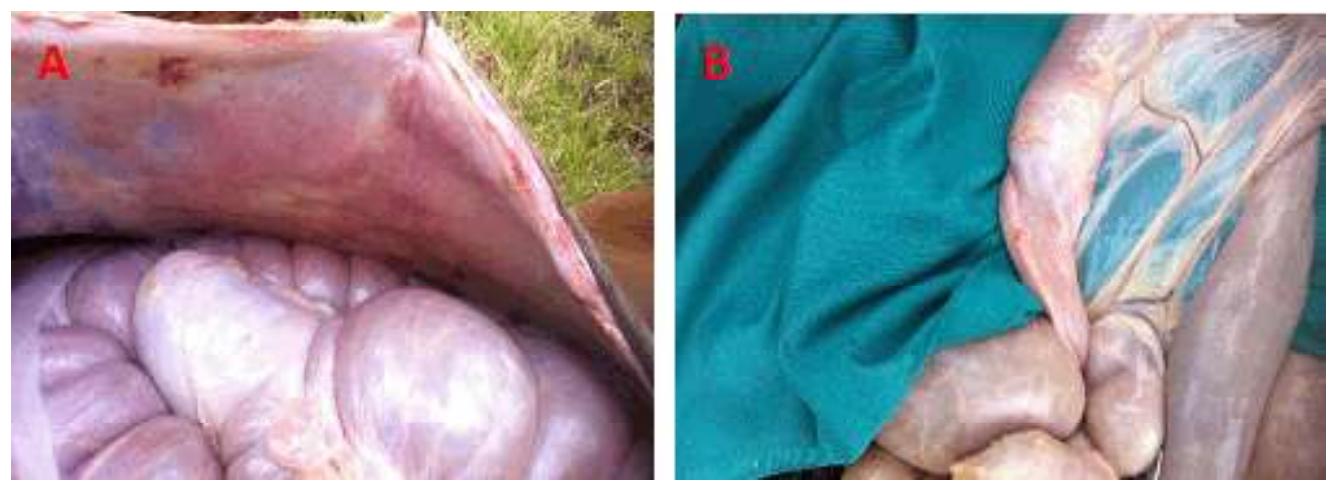

Figura 2. Evaluación macroscópica de la cavidad abdominal. (A) Sin evidencia de alteraciones fisiológicas y ausencia de adherencias; (B) Línea de sutura anastomótica totalmente cicatrizada después de 100 días del posoperatorio
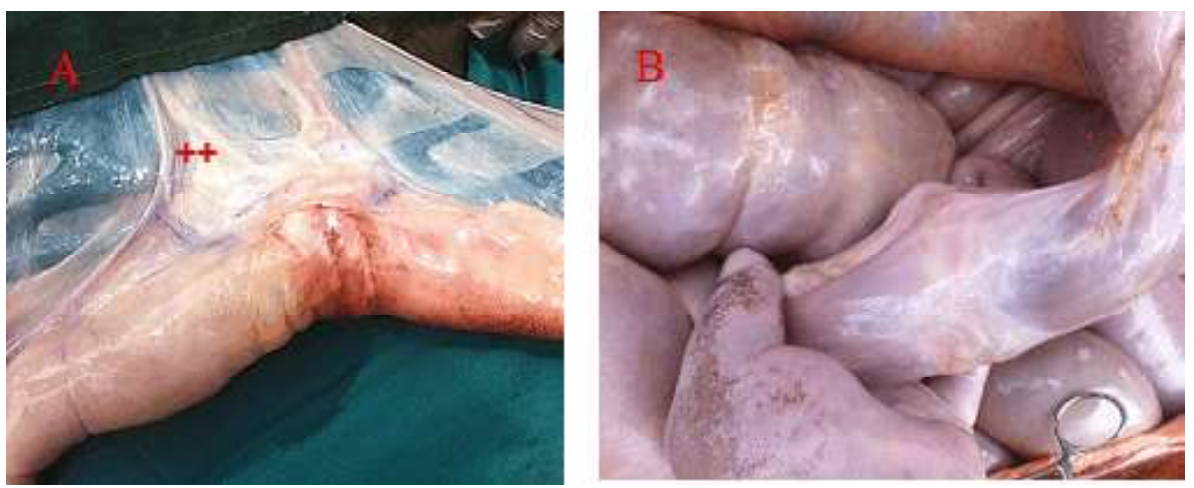

Figura 3. Vista macroscópica de la anastomosis intestinal revestida con xenoinjerto de pericardio bovino a los 15 y 100 días del posoperatorio. A) Yeyuno equino con xenoinjerto de pericardio bovino, de color blanquecino, con una conformación anastomótica en «V» propia de la técnica quirúrgica, que aísla la línea de sutura, donde resalta la hiperemia intestinal fuera del xenoinjerto a los 15 días del posoperatorio. Se evidencia la buena cicatrización del mesenterio $(++)$. La zona anastomótica se encuentra totalmente revestida por tejido mesotelial y el xenoinjerto se ve fusionado al intestino. B). La luz intestinal de la zona anastomótica se observa más grande en comparación al resto del intestino a los 100 días del posoperatorio, perdiendo la forma de «V» inicial.

reacción inflamatoria mucho más contundente debida al hilo de sutura (Figura 6). En cambio, a los 100 días del posoperatorio, la serosa se nota delgada, sin presencia de infiltración inflamatoria en las capas del intestino, aunque se mantenía un infiltrado inflamatorio en la zona del hilo de sutura, indicando que el pericardio bovino sin tratar se adapta mejor al organismo que el ácido poliglicólico (Figura 7). Los resultados demuestran que el re- vestimiento con xenoinjerto de pericardio bovino de la anastomosis término-terminal es viable.

\section{Discusión}

Se resalta la viabilidad de la técnica quirúrgica de anastomosis término-terminal yeyunal con un solo patrón de sutura simple 


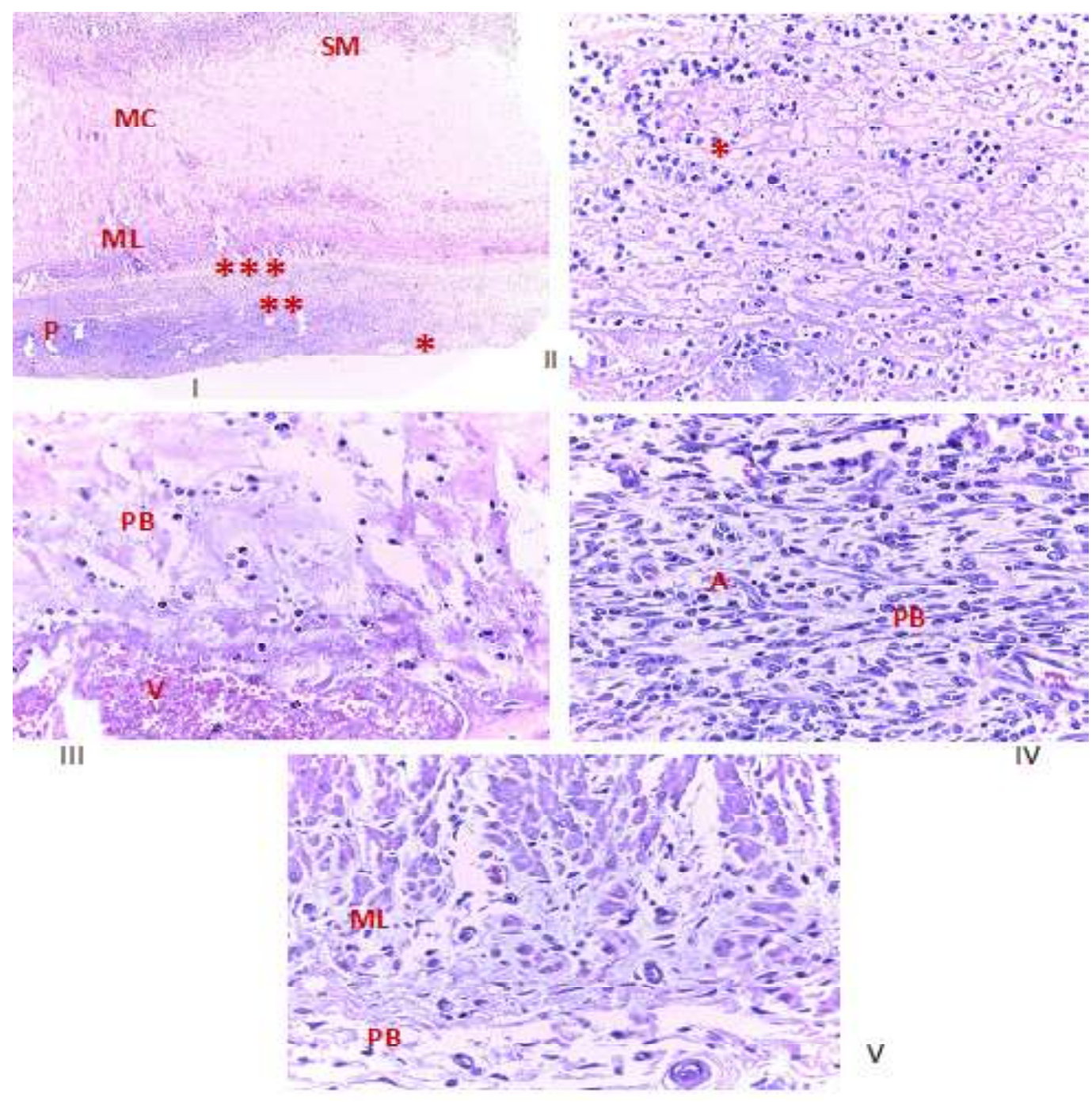

Figura 4. Histopatología de la anastomosis intestinal revestida con xenoinjerto de pericardio bovino a los 15 días del posoperatorio. I). No hay diferencias entre la serosa y el pericardio bovino. Se halla una densa capa serosa compuesta por abundantes neutrófilos en una malla de fibrina (*); el estrato subyacente compuesto por una densa trama de fibrina con leve número de neutrófilos y vasos venosos $(* *)$. La capa siguiente formada por un denso tejido de granulación con abundantes fibroblastos acompañada por colágeno y múltiples angioblastos $(* * *)(\mathrm{H} \& \mathrm{E}$, $4 X)$. II) Se observa una densa capa serosa (acercamiento de I señalado con *) compuesta por un exudado de abundantes neutrófilos en diversos grados de degeneración (H\&E, 40X). III) PB Estrato subyacente (acercamiento de I señalado con $* *$ ) compuesto por una trama de fibrina más densa con leve número de neutrófilos en variables grados de degeneración y vasos venosos ingurgitados por glóbulos rojos. Ausencia de bacterias (H\&E, 40X). IV) Capa formada por un denso tejido de granulación (acercamiento de I señalado con ***), con abundantes fibroblastos jóvenes que se dirigen en varias direcciones y se acompañan por colágeno, algunos neutrófilos y escasos linfocitos y múltiples angioblastos que logran tomar contacto con la capa muscular del intestino. Las capas del intestino aparentemente normales (H\&E, 40X). (V) Tejido de granulación tomando contacto con la capa muscular lisa intestinal (H\&E, 40X). (ML) Capa muscular longitudinal, (MC) Capa muscular circular, (SM) Capa submucosa, (M) Capa mucosa, (PB) Xenoinjerto de pericardio bovino, (V) Vasos sanguíneos, (A) Angioblastos 

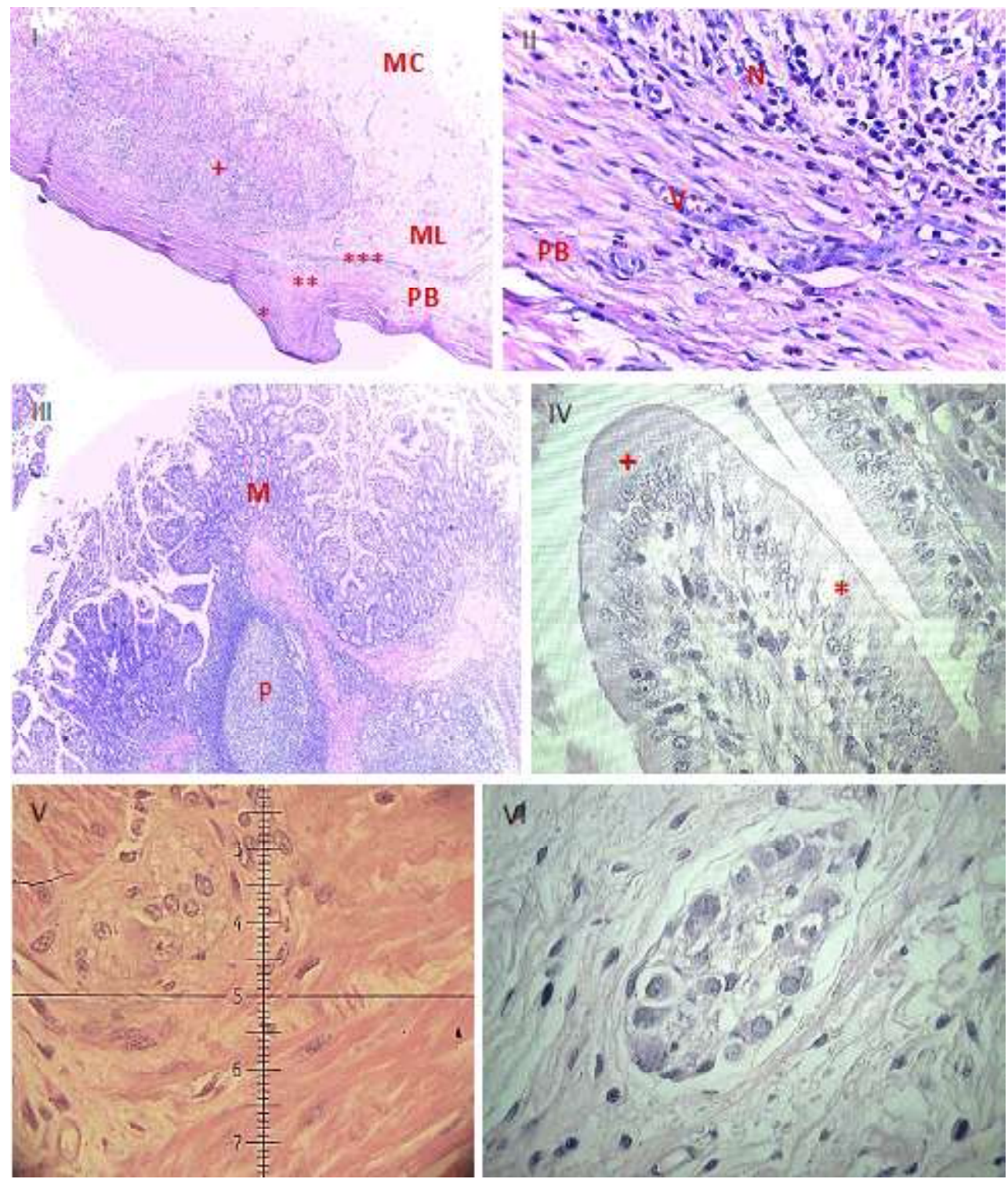

Figura 5. Histopatología de la anastomosis intestinal revestida con xenoinjerto de pericardio bovino a los 100 días del posoperatorio. I) La capa serosa muy delgada $(*)$ y la subserosa con una densa capa de tejido conectivo fibroso $(* *)$ y más subyacentemente una reacción inflamatoria crónica con escasos neutrófilos $(* * *)$; reacción inflamatoria en capa muscular con escasos neutrófilos y un denso tejido conectivo fibroso vascularizado como respuesta al hilo de sutura (+) (H\&E, 40X). II) En el lugar del xenoinjerto se observa una reacción inflamatoria con escasos neutrófilos y un denso tejido conectivo fibroso vascularizado (H\&E, 40X). III) Mucosa aparentemente normal y submucosa con placas de Peyer en hiperplasia folicular linfoide (H\&E, 4X). IV) Microvellosidad yeyunal aparentemente normal; $(+)$ enterocitos, $(*)$ células caliciformes de núcleos conservados con una distribución normal (H\&E, 40X). V y VI) Plexos de Auerbach (izquierda) entre saludables células musculares y plexo de Meissner (derecha) a nivel de la submucosa, en ambos casos con ausencia de reacción inflamatoria (40X). (ML) Capa muscular longitudinal, (MC) Capa muscular circular, (PB) Xenoinjerto de pericardio bovino, (M) Mucosa, (P) Placa de Peyer, (V) Vasos sanguíneos, (N) Neutrófilos 


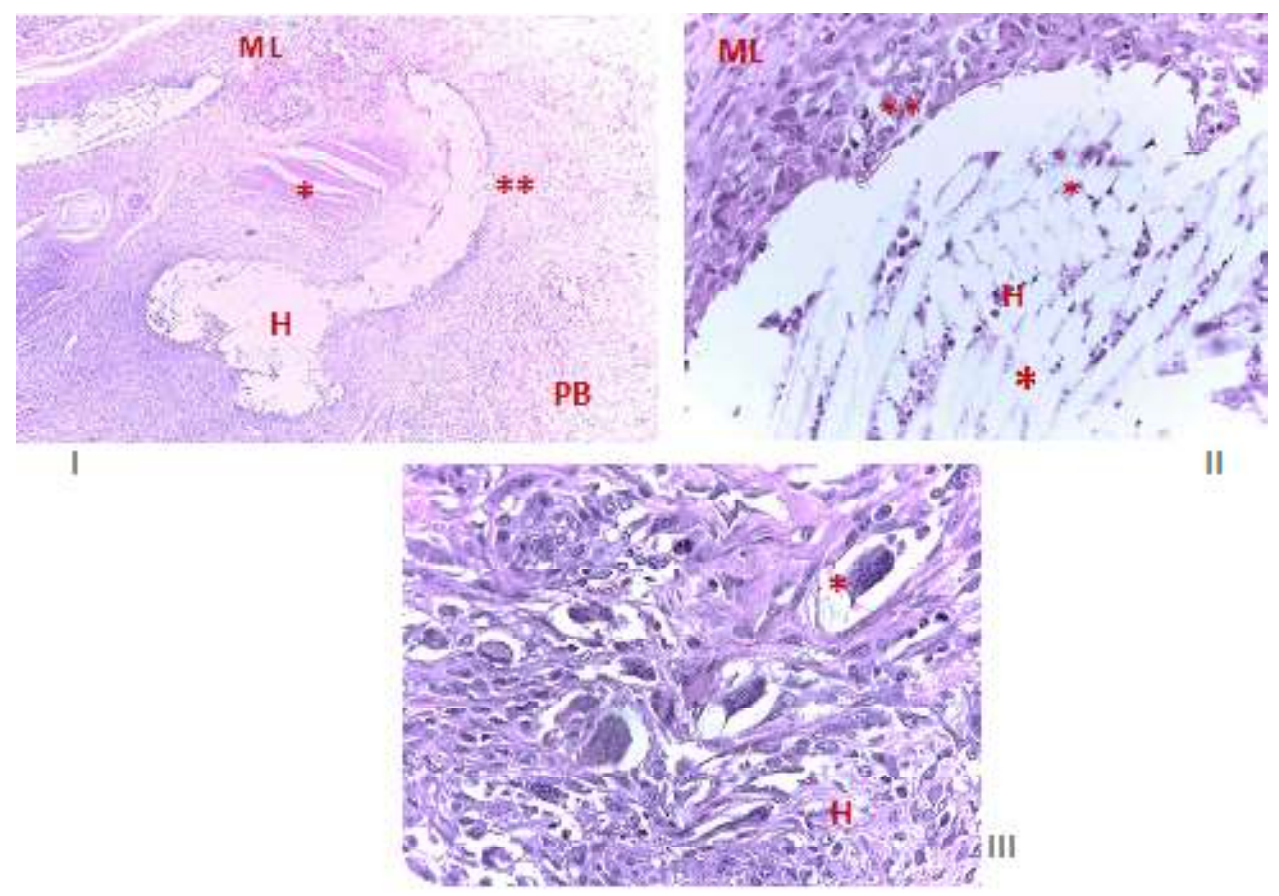

Figura 6. Histopatología del efecto del hilo de sutura en la anastomosis intestinal revestida con xenoinjerto de pericardio bovino a los 15 días del posoperatorio. I) Se observa una estructura compuesta por numerosas elongaciones tipo tubular hialinas entre las capas muscular y del tejido de granulación que se rodean de neutrófilos $(*)$; en la periferia de estas estructuras hay una reacción inflamatoria compuesta por células gigantes $(* *)$ a cuerpo extraño con numerosos macrófagos y leve número de neutrófilos (H\&E, 4X). II) Cuerpo extraño compatible con hilo de sutura, tomando contacto con la capa muscular lisa y compuesto por fibras paralelas hialinas rodeadas por neutrófilos $\left(^{*}\right)$ en varios grados de degeneración, con estructuras rodeadas de un exudado compuesto por macrófagos y células gigantes $(* *)(\mathrm{H} \& \mathrm{E}, 40 \mathrm{X})$. III) Acercamiento de II. Foco de reacción inflamatoria granulomatosa compuesta por abundantes células gigantes (*) rodeando a restos de hilos de sutura, acompañados de escaso número de macrófagos y colágeno entre las células inflamatorias (H\&E, 40X). (PB) Xenoinjerto de pericardio bovino, (ML) Capa muscular longitudinal, (H) Hilo de sutura - ácido poliglicólico 3-0

discontinuo en tres capas. Las suturas que provocan eversión o inversión retrasan la cicatrización del intestino y pueden provocar la formación de estenosis (Fossum, 2009); así mismo, la tasa de mortalidad y la presencia de cólicos en el posoperatorio son mayores en caballos con anastomosis latero-lateral (Proudman et al., 2007), a lo que se suma el aporte de Davis y Rivadeneira (2013), quie- nes señalan que la necrosis de la anastomosis ocurre dentro de 48 horas de la cirugía cuando la saturación de oxígeno disminuye a menos del $30 \%$ en la línea de sutura.

Con relación al manejo del pericardio bovino, Santos et al. (2013) enfatizan la necesidad de tomar precauciones especiales en su manejo, debido a que puede ser una fuen- 

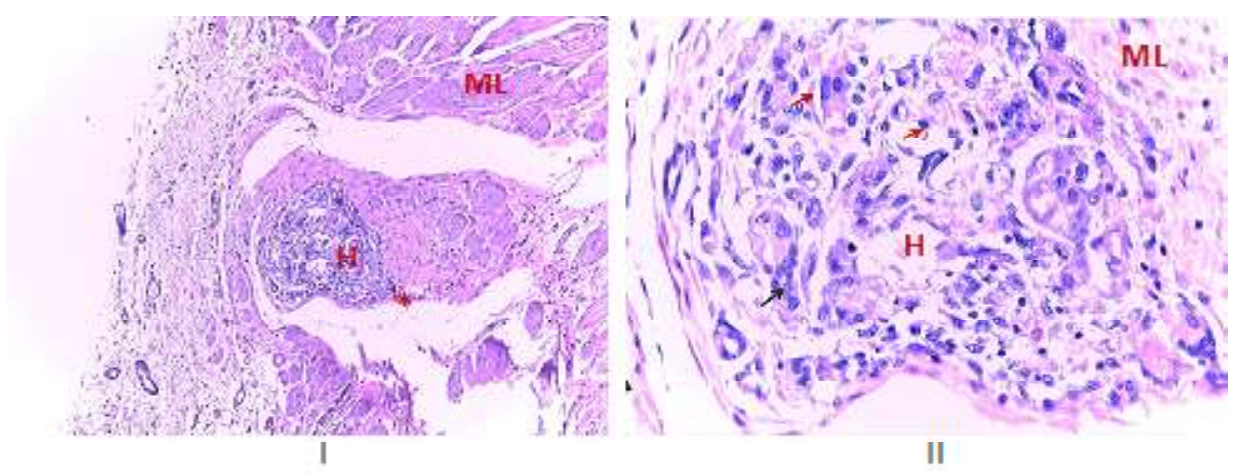

Figura 7. Histopatología del efecto del hilo de sutura en la anastomosis intestinal revestida con xenoinjerto de pericardio bovino a los 100 días del posoperatorio (H\&E, 40X). I) Hilo de sutura. Se observa un foco de células inflamatorias tipo granulomatosas conformadas por moderado número de células gigantes multinucleadas $(*)$ entre la capa muscular. II) Acercamiento de II. Las flechas rojas señalan restos del cuerpo extraño (hilo de sutura) y la flecha negra señala una célula gigante multinucleada (H\&E, 40X). (ML) Capa muscular longitudinal, (H) Hilo de sutura - ácido poliglicólico 3-0

Cuadro 1. Caracterización de los cambios histopatológicos ${ }^{1}$ debidos al revestimiento con xenoinjerto de pericardio bovino no tratado en la anastomosis yeyunal equina a los 15 y 100 días del posoperatorio

\begin{tabular}{clcccccc}
\hline Equino & Variable & $\begin{array}{c}\text { Días } \\
\text { pos- } \\
\text { cirugía }\end{array}$ & Flogosis & Fibrosis & $\begin{array}{c}\text { Estado de } \\
\text { infiltración en } \\
\text { revestimiento }\end{array}$ & $\begin{array}{c}\text { Estado de } \\
\text { mucosa }^{2}\end{array}$ & $\begin{array}{c}\text { Estado } \\
\text { de } \\
\text { serosa }^{3}\end{array}$ \\
\hline 1 & $\begin{array}{l}\text { Anastomosis } \\
\text { de yeyuno }\end{array}$ & 15 & 3 & 2 & 3 & $\mathrm{~N}$ & $\mathrm{E}$ \\
2 & $\begin{array}{l}\text { Zona del } \\
\text { hilo de }\end{array}$ & 15 & 0 & 0 & 0 & $\mathrm{~N}$ & $\mathrm{~N}$ \\
& sutura & 100 & 4 & 3 & - & - & - \\
\hline
\end{tabular}

${ }^{1}$ Escala de puntuación de 0 a 4 ( $0=$ ausente, $1=$ mínima, $2=$ moderada, $\left.3=\operatorname{distintiva,~} 4=\operatorname{severa}\right) ;{ }^{2} \mathrm{~N}$ = normal, $\mathrm{E}=$ erosionada; ${ }^{3} \mathrm{~N}=$ normal, $\mathrm{E}=$ engrosada; según Testini et al. (2014), adaptado para este estudio; (-) no pudo ser evaluado

te potencial de transmisión de enfermedades infecciosas. Sin embargo, en el presente estudio se utilizó una hora después de haber sido extraído, sin contar con precauciones especiales para evitar posibles transmisiones, no habiéndose detectado infecciones evidentes, aunque claro está, el peligro de transmisión de enfermedades infecciosas debe ser evitado.
Los resultados de este estudio indican que el xenoinjerto de pericardio bovino no tratado es una buena opción para evitar las fallas anastomóticas del intestino delgado en el equino, garantizando el proceso de remodelación cicatricial, evitando la falla anastomótica (Telem et al., 2010; Testini et al., 2014). Por otro lado, las perforaciones o fístulas podrían manifestarse con bajo grado 
de cólico (Freeman, 2012), representando mayor dificultad durante el manejo postoperatorio y falla en el pronóstico. Los resultados obtenidos son positivos frente al reporte de Hansen y Monnet (2013), quienes señalan que aún no se tienen evidencias objetivas para el uso de los métodos de revestimiento con ácido poliglicólico, injerto peritoneal y selladores de fibrina.

En la evaluación macroscópica del yeyuno durante la relaparotomía no se halló contracción ni desplazamiento del xenoinjerto; encontrándose más bien, fijado a la zona anastomótica, al igual que en los reportes de Testini et al. (2014). No obstante, otros reportes describen que el refuerzo con vellones de colágeno tratado puede causar obstrucción intestinal, dando como resultados fenómenos de contracción y desplazamiento de las prótesis (Hoeppner et al., 2009; Schreinemacher et al., 2011).

Se conoce que la formación de adherencias se produce principalmente entre los días 7 y 10 de la cirugía (Gorvy et al., 2008) y que para caracterizar la magnitud de las adherencias y órganos implicados se requiere ingresar a la cavidad peritoneal (Siobhan, 2014), de allí que se hizo la segunda laparatomía en dos de los caballos intervenidos. En la evaluación macroscópica no se encontró presencia de adherencias ligeras, fijas, ni sólidas, lo que repercutió en la ausencia del cólico posoperatorio por fallas quirúrgicas. Sin embargo, se reporta que deficiencias intestinales pueden producirse como resultado de la inflamación intestinal o incluso en lugares distantes al lugar de la disfunción intestinal (Tuan et al., 2006; Tappenden, 2008). También es importante considerar la posible presencia de adherencias congénitas en algunos pacientes (Liakakos et al., 2001), que no deberían relacionarse con la cirugía abdominal.

En este estudio, si bien es cierto, no se evaluó la actividad mieloeléctrica de la zona anastomótica, se puede decir que la actividad mecánica y el tránsito gastrointestinal fueron normales después del periodo de 100 días de convalecencia de los equinos, dado que llegaron a obtener un comportamiento alimenticio normal.

El pericardio bovino usado en esta investigación no fue sometido a tratamiento alguno, a diferencia del utilizado por Testini et al. (2014), que junto a otros investigadores indican que el procesamiento del pericardio bovino con glutaraldehído sirve para evitar antígenos celulares, iones de calcio en la circulación y fosfolípidos presentes en el tejido nativo, los cuales son predominantemente responsables de la calcificación, degeneración y falla de los tejidos implantados como un microambiente natural para la migración de células progenitoras (Mathapati et al., 2013). Sin embargo, los exámenes histopatológicos realizados en los dos equinos no evidenciaron calcificación ni degeneración del colágeno, ni mucho menos inviabilidad (figuras 4-5).

Por otro lado, McDade et al. (2013) reportan que los macrófagos se activan para liberar metaloproteinasas de matriz de colágeno, producto del reclutamiento de células fagocíticas responsables de la degeneración estructural de las bioprótesis tratadas con glutaraldehído. En este estudio se evidenció presencia de macrófagos en el tejido a los 15 días del posoperatorio, pero denotándose ausencia de macrófagos a los 100 días, lo que demuestra la buena adaptabilidad y viabilidad del xenoinjerto implantado (Cuadro 1). Así mismo, mencionan que las células U937 son predictivas de la respuesta a los biomateriales de los macrófagos derivados de los monocitos, los cuales ya diferenciados en el pericardio bovino descelularizado con glutaraldehído, alteran morfológicamente su membrana y disminuyen su actividad enzimática celular, con un aumento de la liberación de citoquinas proinflamatorias; sin embargo, esto no se evidenció en esta investigación. No obstante, es importante recalcar que la inflamación es una respuesta homeostática y su objetivo es limi- 
tar la entrada de materiales extraños al organismo y de facilitar la reparación (Martin y Wallace, 2006), aunque la naturaleza de la inflamación depende de la etiología subyacente (Valenciano y Cowell, 2014).

Los hallazgos encontrados alrededor del hilo de sutura (Figuras 6 y 7) evidencian un gran infiltrado inflamatorio compuesto por células gigantes, coincidiendo con Coleman et al. (1974), y numerosos macrófagos con leve número de neutrófilos degenerados ante la presencia del ácido poliglicólico 3-0 usado como hilo de sutura durante el revestimiento con xenoinjerto de pericardio bovino. Esos hallazgos sugieren realizar futuras investigaciones sobre la biocompatibilidad del hilo de sutura, porque incluso a los 100 días del posoperatorio se comprobaron restos de ácido poliglicólico con procesos inflamatorios, siendo lo contrario en el xenoinjerto.

\section{Conclusiones}

- El xenoinjerto de pericardio bovino no tratado, como revestimiento de la anastomosis término-terminal yeyunal equina, es viable porque garantiza el proceso de remodelación cicatricial, no se contrae ni se desplaza de la línea de sutura, evitando peritonitis, estenosis, abscesos y fugas anastomóticas, ni genera adherencias en la cavidad abdominal.

- El xenoinjerto de pericardio bovino no tratado, como revestimiento de la anastomosis término-terminal yeyunal equina, demuestra buena adaptabilidad como microambiente natural para células progenitoras encargadas de la remodelación cicatricial; aunque conlleva a una moderada flogosis y fibrosis que después del periodo de convalecencia desaparecen progresivamente, sin observarse calcificación ni degeneración de colágeno.

\section{Literatura Citada}

1. Aguilar C, Delgado A, Chavera A, Cueva M. 2018. Anastomosis yeyunal término-terminal con xenoinjerto de pericardio bovino en equinos. Rev Inv Vet Perú 29: 106-119. doi: 10.15381/ rivep.v29i1.14190

2. Alonso JM, Garcia Alves AL, Watanabe MJ, Rodrigues CA, Hussni CA. 2014. Peritoneal response to abdominal surgery/ : the role of equine abdominal adhesions and current prophylactic strategies. Vet Med Int 2014, Article ID 279730. doi: 10.1155/2014/279730

3. Berthoud HR. 2008. Vagal and hormonal gut-brain communication: from satiation to satisfaction. Neurogastroent Motil 20: 64-72. doi: 10.1111/j.13652982.2008.01104.x

4. Bladon BM, Hillyer MH. 2000. Effect of extensive ileal resection with a large resulting mesenteric defect and stapled ileal stump in horses with a jejunocaecostomy: a comparison with other anastomotic techniques. Equine Vet J 32: 52-58. doi: 10.1111/j.2042-3306.2000.tb05334.x

5. Claunch K, Mueller P. 2012. Treating intra-abdominal adhesions: The surgeon's dilemma. Equine Vet Educ 24: 552-555. doi: 10.1111/j.2042-3292.2012.00438.x

6. Coleman DL, King X, Andrade D. 1974. The foreign body reaction: a chronic inflammatory response. J Biomed Mater Res B 8: 199-211. doi: 10.1002/jbm.820080503

7. Davis B, Rivadeneira DE. 2013. Complications of colorectal anastomoses: leaks, struactures, and bleeding. Surg Clin N Am 93: 61-87.

8. Fossum TW. 2009. Cirugía en pequeños animales. $3^{\circ}$ ed. España: Elsevier. $1632 \mathrm{p}$

9. Freeman D. 2012. Small intestine. In: Equine surgery. $4^{\text {th }}$ ed. Canada: Elsevier. p. 416-453. 
10. Furness JB. 2008. The enteric nervous system/ : normal functions and enteric neuropathies. Neurogastroent Motil 20: 32-38. doi: $0.1111 /$ j.1365-2982.2008.01094.x

11. Gelberg HB. 2014. Comparative anatomy, physiology, and mechanisms of disease production of the esophagus, stomach, and small intestine. Toxicol Pathol 42: 54-66. doi: 10.1177/0192623313518113

12. Gorvy DA, Barry G, Proudman CJ. 2008. Intra-abdominal adhesions in horses: a retrospective evaluation of repeat laparotomy in 99 horses with acute gastrointestinal disease. Vet J 175: 194-201. doi: 10.1016/j.tvj1.2007.02.016

13. Hansen LA, Monnet EL. 2013. Evaluation of serosal patch supplementation of surgical anastomoses in intestinal segments from canine cadavers. Am J Vet Res 74: 1138-1141. doi: 10.2460/ ajvr.74.8.1138

14. Hoeppner J, Crnogorac V, Marjanovic G, Jüttner E, Keck T, Weiser HF, Hopt UT. 2009. Small intestinal submucosa for reinforcement of colonic anastomosis. Int J Colorectal Dis 24: 543-550. doi: 10.1007/s00384-009-0637-y

15. Koenig J, Cote N. 2006. Equine gastrointestinal motility - ileus and pharmacological modification. Can Vet J 47: 551-559.

16. König HE, Liebich HG 2011. Anatomía de los animales domésticos: texto y atlas en color. $2^{\circ}$ ed. Madrid: Médica Panamericana. $720 \mathrm{p}$.

17. Li L, Wang N, Jin X, Deng R, Nie S, Sun L, Wu $Q$, et al. 2014. Biodegradable and injectable in situ cross-linking chitosan-hyaluronic acid based hydrogels for postoperative adhesion prevention. Biomaterials 35: 3903-3917. doi: 10.1016/j.biomaterials.2014.01.050

18. Liakakos T, Thomakos N, Fine PM, Dervenis C, Young RL. 2001. Peritoneal adhesions: etiology, pathophysiology, and clinical significance - recent advances in prevention and management.
Digest Surg 18: 260-273. doi: 10.1159/ 000050149

19. Martin GR, Wallace JL. 2006. Gastrointestinal inflammation: a central component of mucosal defense and repair. Exp Biol M 231: 130-137. doi: 10.1177/153537020623100202

20. Mathapati S, Bishi DK, Guhathakurta $S$, Cherian KM, Venugopal JR, Ramakrishna S, et al. 2013. Biomimetic acellular detoxified glutaraldehyde cross-linked bovine pericardium for tissue engineering. Mater Sci Eng 33: 1561-1572. doi: 10.1016/j.msec.2012.12.062

21. Mazzone A, Farrugia G. 2007. Evolving concepts in the cellular control of gastrointestinal motility: neurogastroenterology and enteric sciences. Gastroenterol Clin North Am 36: 499513. doi: 10.1016/j.gtc.2007.07.003

22. McDade JK, Brennan-Pierce EP, Ariganello MB, Labow RS, Lee JM. 2013. Interactions of U937 macrophagelike cells with decellularized pericardial matrix materials: influence of crosslinking treatment. Acta Biomater 9: 7191-7199. doi: 10.1016/j.actbio.2013.02.021

23. Merritt AM, Julliand V. 2013. Gastrointestinal physiology. In: Equine applied and clinical nutrition: health, welfare and performance. Toronto: Elsevier. p 3-32.

24. Proudman CJ, Edwards GB, Barnes J. 2007. Clinical evidence article differential survival in horses requiring end-to-end jejunojejunal anastomosis compared to those requiring side-to-side jejunocaecal anastomosis. Equine Vet $\mathrm{J}$ 39: 181-185. doi: 10.2746/042516407X171354

25. Santos MH, Silva RM, Dumont VC, Neves JS, Mansur HS, Heneine LGD. 2013. Extraction and characterization of highly purified collagen from bovine pericardium for potential bioengineering applications. Mater Sci Eng 33: 790-800. doi: 10.1016/j.msec.2012.11.003 
26. Sasaki N, Yoshihara T. 1998. The effect of motilin on the regulation mechanism of intestinal motility in conscious horses. J Vet Med Sci 61: 167170. doi: $10.1292 /$ jvms.61.167

27. Schreinemacher MH, Bloemen JG, van der Heijden SJ, Gijbels MJ, Dejong CH, Bouvy ND. 2011. Collagen fleeces do not improve colonic anastomotic strength but increase bowel obstructions in an experimental rat model. Int J Colorectal Dis 26: 729-35. doi: 10.1007/s00384-011-1158-z

28. Siobhan M. 2014. Gastrointestinal system. In: Knottenbelt and Pascoe's color atlas of diseases and disorders of the horse. $2^{\text {nd }}$ ed. China: Elsevier. p 1-83.

29. Tappenden KA. 2008. Inflammation and intestinal function: where does it start and what does it mean? JPen-Parenter Enter 32: 648-650. doi: 10.1177/ 0148607108325177

30. Telem DA, Chin EH, Nguyen SQ, Divino $C M$. 2010. Risk factors for anastomotic leak following colorectal- surgery: a case-control study. Arch SurgChicago 145: 371-376. doi: 10.1001/ archsurg. 2010.40

31. Testini M, Gurrado A, Portincasa P, Scacco S, Marzullo A, Piccinni G, Lissidini G, et al. 2014. Bovine pericardium patch wrapping intestinal anastomosis improves healing process and prevents leakage in a pig model. Plos One 9: e86627. doi: 10.1371/journal.pone. 0086627

32. Trigo F, Valero G 2004. Patología general veterinaria. $4^{\circ}$ ed. México: Universidad Autónoma de México. 437 p.

33. Tuan RS, Boland G, Tuli R. 2006. Adult mesenchymal stem cells and cell-based tissue engineering. Arthritis Res Ther 5: 339-361. doi: 10.1186/ar614

34. Valenciano A, Cowell R. 2014. Cowell and Tyler's Diagnostic cytology and hematology of the dog and cat. $4^{\text {th }}$ ed. Duncan L (ed). St. Louis, USA: Elsevier. $608 \mathrm{p}$. 